\title{
Phase-Field Simulation for Non-isothermal Solidification of Al-Cu Binary Alloy
}

\author{
Liu Guangguang ${ }^{a, b}$, Luo Sen ${ }^{a, b *}$, Wang Zhaohui ${ }^{a, b}$, Wang Weiling ${ }^{a, b}$, Zhu Miaoyong ${ }^{a, b}$, \\ Wang Xiaohua ${ }^{c}$ \\ ${ }^{a}$ Key Laboratory for Ecological Metallurgy of Multimetallic Ores, Ministry of Education, Northeastern \\ University, Shenyang, 110819, China \\ ${ }^{b}$ School of Metallurgy, Northeastern University, Shenyang, 110819, China \\ ${ }^{c}$ College of Chemistry, Chemical Engineering and Environmental Engineering, Liaoning Shihua \\ University, Fushun, Liaoning, 11301, China
}

Received: October 19, 2018; Revised: May 13, 2019; Accepted: May 19, 2019

\begin{abstract}
Based on the principle of dilute solution approximation, a phase field model for dendritic growth during the non-isothermal solidification process was proposed by coupling the phase field, concentration field and temperature field, and was adopted to investigate the dendritic growth of Al-Cu binary alloy during the non-isothermal solidification process. Also, the simulations of free dendritic growth in an undercooled melt of $\mathrm{Al}-4.5 \% \mathrm{Cu}$ binary alloy with different perturbation intensity and anisotropy were carried out by the present phase field model. The results show that during the non-isothermal solidification process of $\mathrm{Al}-4.5 \% \mathrm{Cu}$ binary alloy, the dendrite grow into undercooled melt with the solute rejection and latent heat release at the front of solid/liquid interface, and the solute enriches at the dendrite root and high temperature appears at the dendritic growth front. With the increase of perturbation intensity, the dendritic growth becomes more developed and more branches appear. Moreover, the anisotropy coefficient also has a great effect on the dendritic growth, and the growth speed of dendrite increases with the increase of anisotropy coefficient.
\end{abstract}

Keywords: phase-field method, binary alloy, dendritic growth, solute distribution, non-isothermal solidification.

\section{Introduction}

Recently, it has become a popular method to simulate the dendrite growth in the solidification process of steel by numerical method ${ }^{1}$. However, it is difficult to calculate dendrite growth for traditional method, because it must track the solid-liquid interface accurately. The phase field method is a simulation method for describing the evolution of complex interfaces in non-equilibrium state and does not need to track the interface front ${ }^{2}$. Phase field method is usually needed to couple with temperature field, solute field and other external fields to simulate dendrite growth during solidification, thus the process of dendrite growth, solute segregation and other complex process during solidification can be reproduced. The effect of dynamics, perturbation, anisotropy and other factors on microstructure formation also can be studied by phase field method ${ }^{3}$.

The initial purpose of phase-field method is to simulate the dendrite growth of pure matter in undercooled melts, and then Wheeler et al. ${ }^{4,5}$ established the pioneer model (WBM model) for simulating dendritic growth of binary alloys during the solidification process, which was widely adopted to predict the solidification structure of alloys by the following researchers ${ }^{6-8}$. However, the model is required to be performed under the condition of limited interface thickness. Kim et al. ${ }^{9-14}$ also developed a phase field model (KKS model) for alloy solidification, which eliminates the limit of the thin interface thickness, and it was widely adopted to predict the solidification structure of alloys in the past few decades. Ode et al..$^{14}$ used the KKS model to study Ostwald ripening, isothermal dendritic growth of $\mathrm{Fe}-\mathrm{C}$ alloys, based on the dilute solution theory. Zhang et al. ${ }^{15}$ and Niu et al. ${ }^{16}$ studied the effects of undercooling and anisotropy on the primary dendrites morphology of $\mathrm{Fe}-\mathrm{C}$ alloys.

In this work, the phase field model proposed by Kim et al. was adopted to coupled with the solute field and temperature field to simulate the solidification process of Al-Cu alloy under non-isothermal conditions, then the effects of anisotropy and perturbation intensity on dendritic growth were studied.

\section{Phase-field Model}

\subsection{Governing equations for phase field}

Phase field method introduces a phase field variable $\phi$ to represent the phase state of the material in the system. The liquid phase and solid phase are respectively indexed by $\phi=$ 0 and 1 . When $\phi$ is among 0 and 1 , it means the solid-liquid interface. The governing equation for the phase field can be expressed as: 


$$
\frac{\partial \phi}{\partial t}={ }_{-} M \frac{\partial F}{\partial \phi}
$$

where $M$ is the phase field mobility related to the driving force of the interface, $t$ is the time, $F$ is the Helmholtz free energy function, and can be determined by:

$$
F=\int_{V}\left[\frac{1}{2} \varepsilon^{2}\left|\nabla \phi^{2}\right|^{2}+f(\phi, T)\right] d V
$$

where $V$ is the volume, $\varepsilon$ is the gradient energy coefficient setting the scale of the surface tension, $T$ is the temperature, $f(\phi, T)$ is the free energy density, which is defined as the sum of the fraction-weighted free energies of liquid and solid phase and an imposed double-well potential and can be determined by

$$
f(\phi, T)=h(\phi) f^{s}+(1-h(\phi)) f^{L}+W g(\phi)
$$

where $f^{s}$ and $f^{L}$ represent the free energy densities of the solid and liquid phases respectively. $g(\phi)$ is a double-well potential, $W$ is its height. The interpolation function $h(\phi)$ represents a normalized area under the potential. They are given by

$$
\begin{gathered}
h(\phi)=\phi^{3}\left(10-15 \phi+6 \phi^{2}\right) \\
g(\phi)=\phi^{2}(1-\phi)^{2}
\end{gathered}
$$

Thus, the governing equations for the phase field is given by

$$
\frac{\partial \phi}{\partial t}=M\left[\varepsilon^{2}(\theta) \nabla^{2} \phi-f_{\phi}\right]
$$

where $f_{\phi}$ is the result of equation (3) for solving partial differential of $\phi$, it can be given by

$$
f_{\phi}=-\frac{R T}{V_{m}} h^{\prime}(\phi) 1 n\left[\frac{\left(1-c_{l}\right)\left(1-c_{s}^{e}\right)}{\left(1-c_{s}\right)\left(1-c_{l}^{e}\right)}\right]+W g^{\prime}(\phi)
$$

where $h^{\prime}(\phi)$ and $g^{\prime}(\phi)$ respectively represent the first partial derivatives by $\phi . c_{l}$ and $c_{s}$ represent the concentrations of the liquid phase and the solid phase respectively, $c^{e}{ }_{l}$ and $c^{e}{ }_{s}$ are the equilibrium concentrations of the liquid phase and the solid phase respectively.

In order to take the anisotropy of solid/liquid interface into consideration, the parameter $\varepsilon(\theta)$ related to the interface energy is expressed as:

$$
\varepsilon(\theta)=\varepsilon(1+v \cos (k \theta))
$$

where $k$ is 4 for the four-hold anisotropy, $v$ represents anisotropic magnitude. $\theta$ is the angle between normal direction of interface and the reference axis (usually is $x$-axis).

\subsection{Governing equations for solute diffusion}

The governing equation for the concentration field can be expressed as:

$$
\frac{\partial c}{\partial t}=\nabla\left[\frac{D(\phi)}{f_{c c}} \nabla f_{c}\right]
$$

where $c$ represents the concentration of the solute, $D_{i}(\phi)$ represents the diffusion coefficient of the solute. $f_{c}$ and $f_{c c}$ represent the first and second partial derivatives of $f$ by $c$, respectively. According to the dilute solution approximation, the following expressions can be derived:

$$
\begin{aligned}
& c=h(\phi) c_{s}+(1-h(\phi)) c_{1} \\
& \mu^{s}\left(c_{s}(x, t)\right)=\mu^{1}\left(c_{1}(x, t)\right)
\end{aligned}
$$

where the $\mu_{s}$ and $\mu_{l}$ in the formula represent the chemical potentials of solid phase and liquid phase respectively.

\subsection{Governing equations for temperature field}

The temperature field is also coupled in the calculation. The effect of latent heat release on temperature field is considered in this work. The influence of latent heat is estimated by the sum of the variation of phase field variables in the corresponding temperature field meshes. So the thermal diffusion equation can be expressed as:

$$
\frac{\partial T}{\partial t}=\alpha \nabla^{2} T+\frac{L}{c_{p}} h^{\prime}(\phi) \frac{\partial \phi}{\partial t}
$$

where $\alpha$ is the thermal diffusivity, $T$ is the temperature, $L$ is the latent heat, $c_{p}$ is specific heat. And the thermal diffusivity can be calculated by:

$$
\alpha=\frac{k}{\rho C_{p}}
$$

where $\rho$ is the density and $C_{p}$ is the specific heat.

\section{Calculation Process}

In the present study, numerical simulations were performed for the $\mathrm{Al}-4.5 \% \mathrm{Cu}$ binary alloy with a uniform melt temperature $900 \mathrm{k}$ (33.3 K undercooling) in a domain size of $12 \mu \mathrm{m} \times 12 \mu \mathrm{m}$. The top, left, and right boundaries are insulated, the heat in the calculation domain is extracted to the environment through the bottom, and the temperature at the bottom boundary is set to be room temperature $(300 \mathrm{~K})$. The calculation domain is divided into $1200 \times 1200$ cells and each cell is initialized with characteristic parameters, such as, nominal composition, temperature, phase state, etc. We assume a nuclei with the preferred growth orientation parallel to the coordinate axes nucleates at the center of the calculation domain at the beginning of the simulation. Without another specification, the model configuration is used in the following simulation cases.

In order to simulate the perturbation of the interface at the actual solidification process, random perturbation is added in this work. It was given as follow: 


$$
\frac{\partial \phi}{\partial t}=\frac{\partial \phi}{\partial t}+16 g(\phi) \chi \bar{\omega}
$$

where $\chi$ is a random number between -1 and $1 . \bar{\omega}$ is the perturbation intensity factor.

In the calculation, the explicit finite difference method is used to solve the discrete solution of the phase field equation , solute diffusion equation and thermal diffusion equation. The calculation process is implemented in terms of Visual $\mathrm{C}++$, and visualization of the calculation results is treated by the commercial software Tecplot. The thermophysics parameters of $\mathrm{Al}-\mathrm{Cu}$ binary alloy adopted in the present study are listed in the Table 1.

\section{Results and Discussion}

\subsection{Effect of perturbation intensity}

\subsubsection{Dendrite morphology}

Figure 1 shows the snapshot of dendrite morphology in an undercooled melt of Al-Cu binary alloy with different perturbation intensities after $4.7 \times 10^{-5} \mathrm{~s}$. It can be seen that with the increase of perturbation intensity, the solid/liquid interface of the primary trunk becomes more unstable, and the secondary dendrite arm begins emitting from the primary trunk. The secondary dendrite arm growth has undertaken a great competition, and some secondary dendrite is inhibited by the well-developed secondary dendrite. Especially for the secondary dendrite arms at the root of primary dendrite trunk, they suffers from a great competition, and thus they are less developed. Also, with the increase of perturbation intensity, the dendrite grows more developed and the tertiary dendrite arms appears for the perturbation intensity of 0.02 . Therefore, for the higher perturbation intensity, the dendrite grows with more complicated arm branching.

\subsubsection{Solute and temperature distribution}

Figure 2 shows the copper distribution in an undercooled melt of Al-Cu binary alloy with different perturbation intensities after $4.7 \times 10^{-5} \mathrm{~s}$ : (a) 0 , (b) 0.01 , and (c) 0.02 . It can be seen that the distribution of solute is consistent with that of dendrite growth. The lowest concentration of $\mathrm{Cu}$ in the dendrite center is due to the undercooling caused by the curvature effect of dendrite tip during solidification, which decreases the solidus temperature, and the diffusion rate of solute in the solid phase is much smaller than the growth rate of dendrite. $\mathrm{Cu}$ enrichment occurs at the solidliquid interface of dendrite growth, which is due to solute redistribution during solidification. The concentration of $\mathrm{Cu}$ in solid phase is lower than the initial concentration, and the
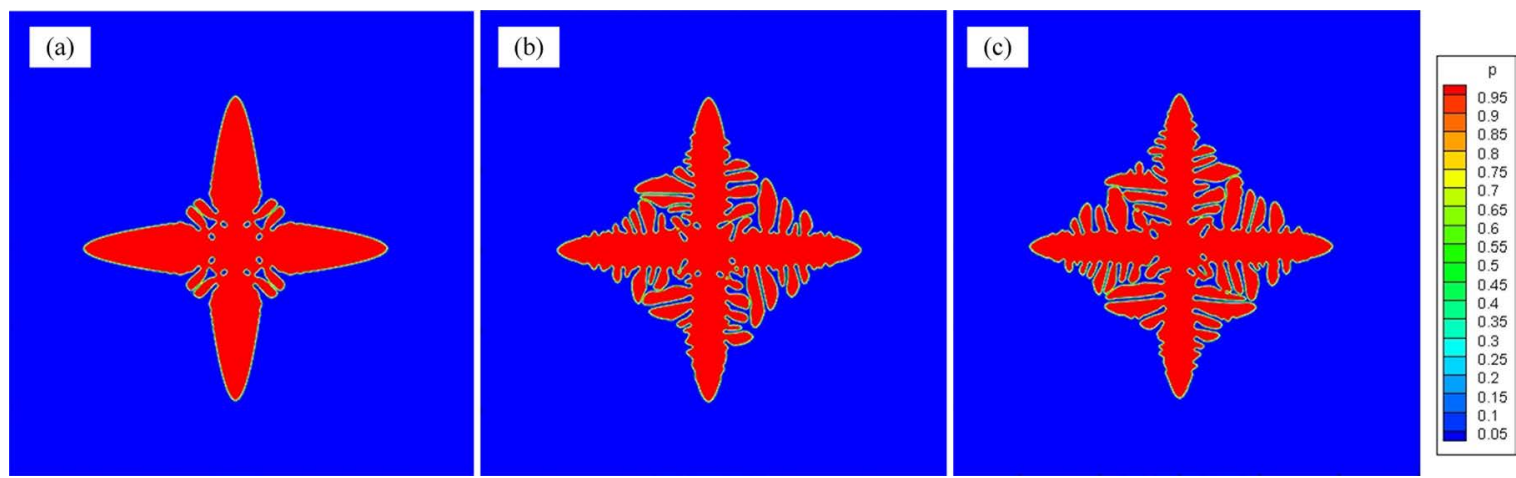

Figure 1. Snapshot of dendrite morphology in an undercooled melt of Al-Cu binary alloy with different perturbation intensities after $4.7 \times 10^{-5} \mathrm{~s}$ : (a) 0 , (b) 0.01 , and (c) 0.02

Table 1. Thermophysics parameters of Al-Cu alloy.

\begin{tabular}{cc}
\hline Parameter & Values \\
\hline Equilibrium distribution coefficient, $k_{e}$ & 0.14 \\
Liquidus slope, $m_{e}(\mathrm{~K} / \mathrm{mol})$ & 640 \\
Solute diffusivity in liquid phase,$D_{l}\left(\mathrm{~m}^{2} / \mathrm{s}\right)$ & $3 \times 10^{-9}$ \\
Solute diffusivity in solid phase, $D_{s}\left(\mathrm{~m}^{2} / \mathrm{s}\right)$ & $3 \times 10^{-13}$ \\
Interface energy, $\sigma\left(\mathrm{J} / \mathrm{m}^{2}\right)$ & 0.093 \\
Melting point, $\mathrm{T}_{\mathrm{m}}(\mathrm{K})$ & 933.3 \\
Latent heat of melting, $L(\mathrm{~kJ} / \mathrm{kg})$ & 389 \\
Specific heat, $\left.C_{p}(\mathrm{~J} /(\mathrm{kg} \cdot \mathrm{K}))\right)$ & 1130 \\
Thermal conductivity, $k(\mathrm{~W} /(\mathrm{m} \cdot \mathrm{K}))$ & 192.6 \\
Density, $\rho\left(\mathrm{kg} / \mathrm{m}^{3}\right)$ & 2800 \\
\hline
\end{tabular}


diffusion rate of solute in liquid phase is much lower than that of dendrite growth, thus solute enrichment appears at the dendrite front. Figure 3 shows the temperature distribution in an undercooled melt of Al-Cu binary alloy with different perturbation intensities after $4.7 \times 10^{-5} \mathrm{~s}$ : (a) 0 , (b) 0.01 , and (c) 0.02 . It can be seen that the distribution of temperature is also consistent with dendrite growth. The latent heat release during the solidification process makes the temperature higher in the solid phase than that in the liquid phase, and the temperature near the liquid surface is higher than that at the center. At the dendrite tip, the temperature gradient is maximal due to the large interfacial movement rate, and the latent heat release. At the dendrite root, the temperature gradient is minimal due to the small interfacial movement rate. And the maximum temperature in the solid phase decreases with increasing perturbation intensity.

\subsection{Effect of anisotropy}

\subsubsection{Dendrite morphology}

Figure 4 shows the snapshot of dendrite morphology in an undercooled melt of Al-Cu binary alloy with different anisotropies after $4.7 \times 10^{-5} \mathrm{~s}$ : (a) 0.03 , (b) 0.04 , and (c) 0.05 .
It can be seen that with the increase of anisotropy, the dendrite trunk becomes longer, the dendritic growth are developed with more secondary dendrite arms, and the secondary dendrite also grows longer, which indicates that anisotropy can promote the growth of the main dendrite and secondary dendrite. And also the tertiary dendrites appears with the increase of anisotropy, therefore, the anisotropy has an effect on the dendritic growth, and with the increase of anisotropy, the dendrite grows more developed.

\subsubsection{Solute and temperature distribution}

Figure 5 shows the copper distribution in an undercooled melt of Al-Cu binary alloy with different anisotropies after $4.7 \times 10^{-5} \mathrm{~s}$ : (a) 0.03 , (b) 0.04 , and (c) 0.05 . It can be seen that the solute is enriched at the root of primary dendrite trunk and secondary dendrite arm, thus the copper concentration in the interdendritic liquid is higher than that of dendrite arm. The enrichment of $\mathrm{Cu}$ at the front the solid/liquid interface is due to the solute redistribution during solidification process, the concentration of $\mathrm{Cu}$ in the solid phase is lower than the nominal concentration, the solute diffusion velocity in liquid phase is less than that of dendrite growth, and the rejected solute at the solid/liquid interface cannot be fully diffused
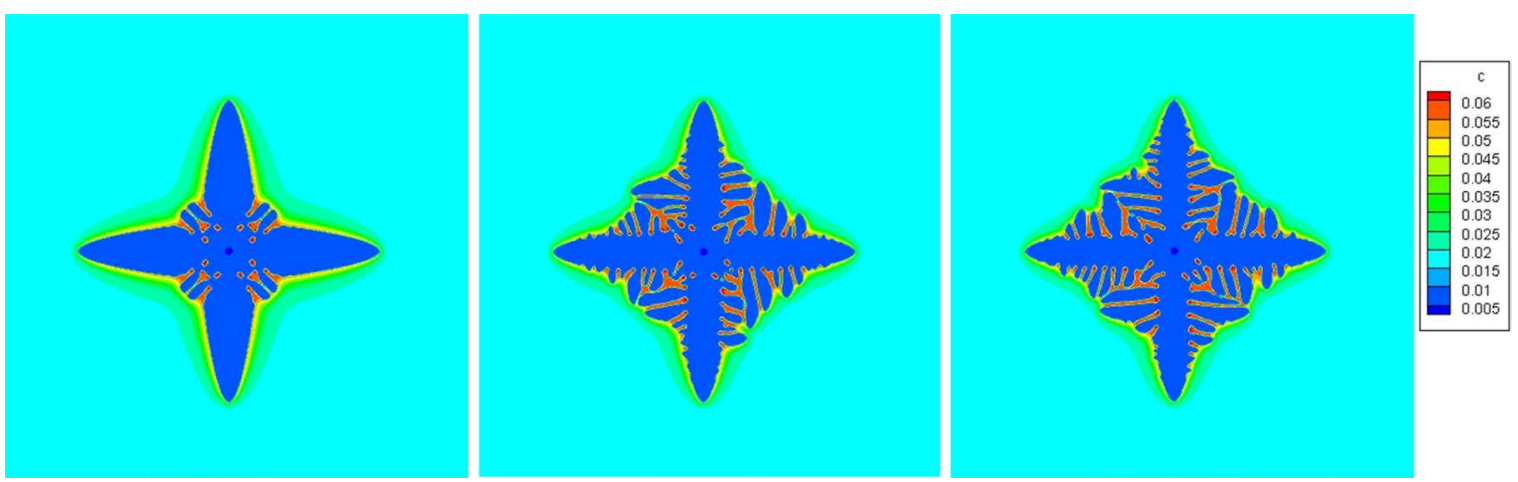

Figure 2. Copper distribution in an undercooled melt of Al-Cu binary alloy with different perturbation intensities after $4.7 \times 10^{-5} \mathrm{~s}$ : (a) 0 , (b) 0.01 , and (c) 0.02
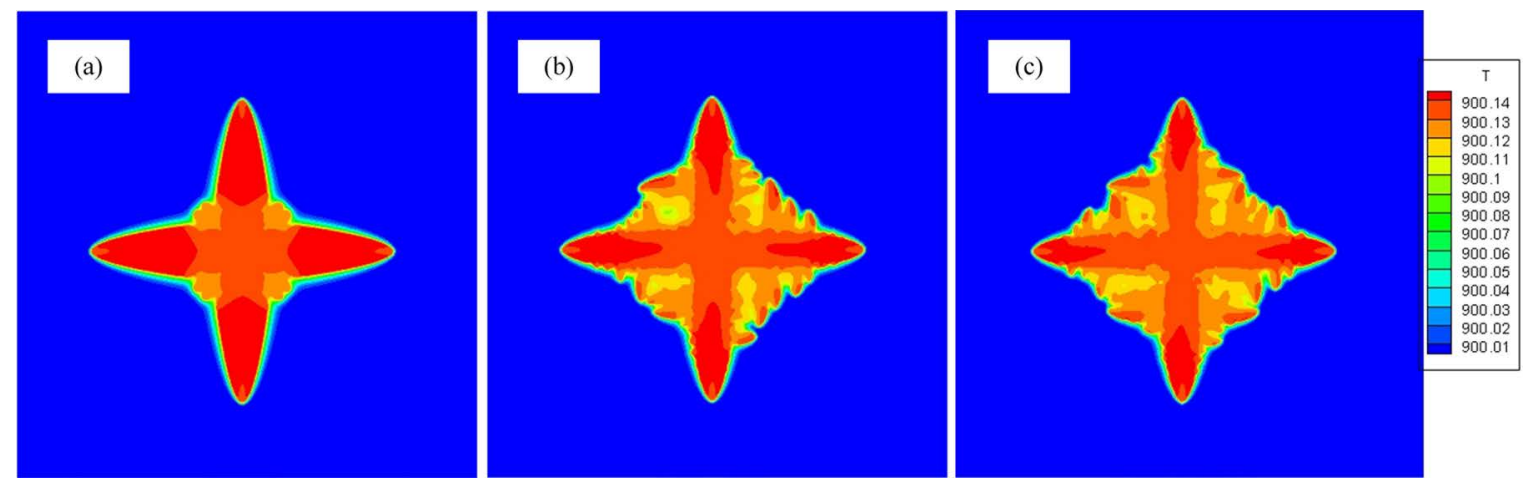

Figure 3. Temperature distribution in an undercooled melt of Al-Cu binary alloy with different perturbation intensities after $4.7 \times 10^{-5} \mathrm{~s}$ : (a) 0 , (b) 0.01 , and (c) 0.02 

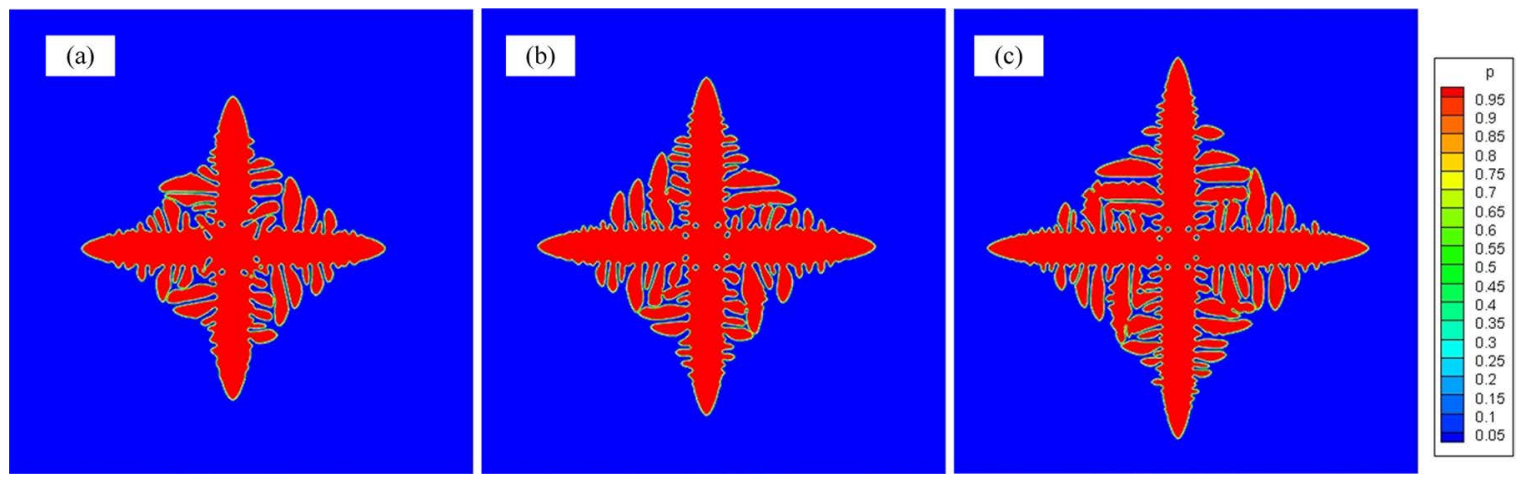

Figure 4. Snapshot of dendrite morphology in an undercooled melt of Al-Cu binary alloy with different anisotropies after $4.7 \times 10^{-5} \mathrm{~s}:(\mathrm{a})$ 0.03 , (b) 0.04 , and (c) 0.05

into the liquid phase, thus solute enrichment appears at the dendritic growth front. Figure 6 shows the temperature distribution in an undercooled melt of Al-Cu binary alloy with different anisotropies after $4.7 \times 10^{-5} \mathrm{~s}$ : (a) 0.03 , (b) 0.04 , and (c) 0.05 . It can be seen that the maximum temperature appears at the dendritic growth front, where the latent heat releases more during non-isothermal solidification process, and its distribution is consistent with the concentration distribution. With the increase of anisotropic intensity, the dendrite grows with more branches, but the solute is still enriched at the root of dendrite and the maximum temperature also appears at the dendritic growth front. Thus, the anisotropic intensity affects the dendritic growth, but it does not has great effects on the concentration field and temperature field.
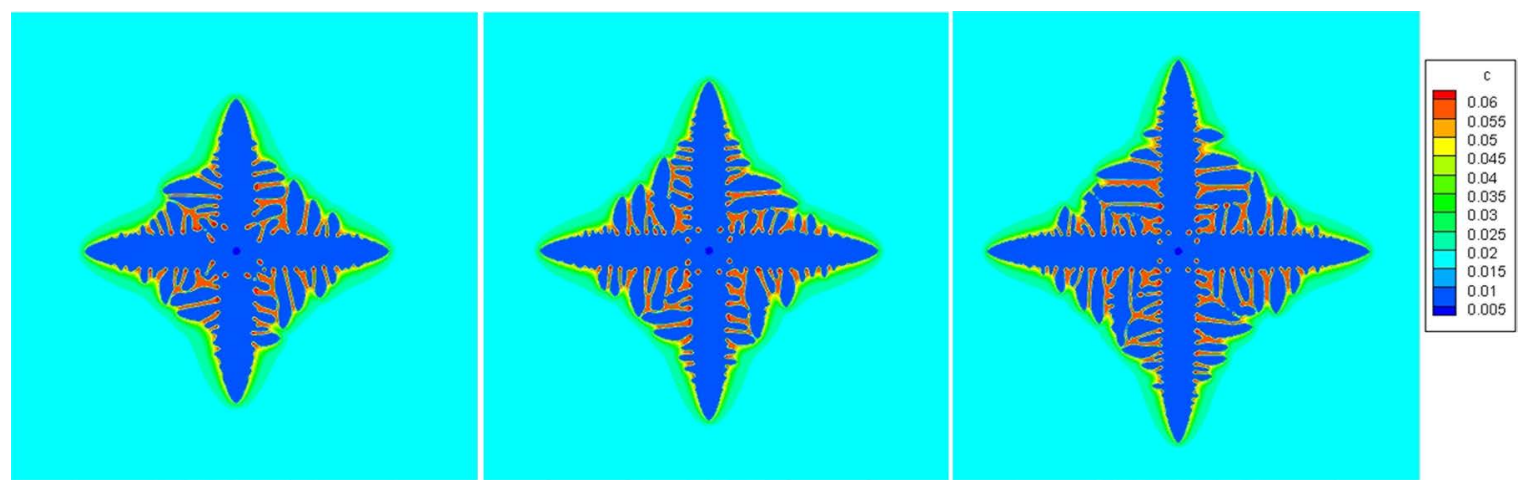

Figure 5. Copper distribution in an undercooled melt of Al-Cu binary alloy with different anisotropies after $4.7 \times 10^{-5} \mathrm{~s}$ : (a) 0.03 , (b) 0.04 , and (c) 0.05
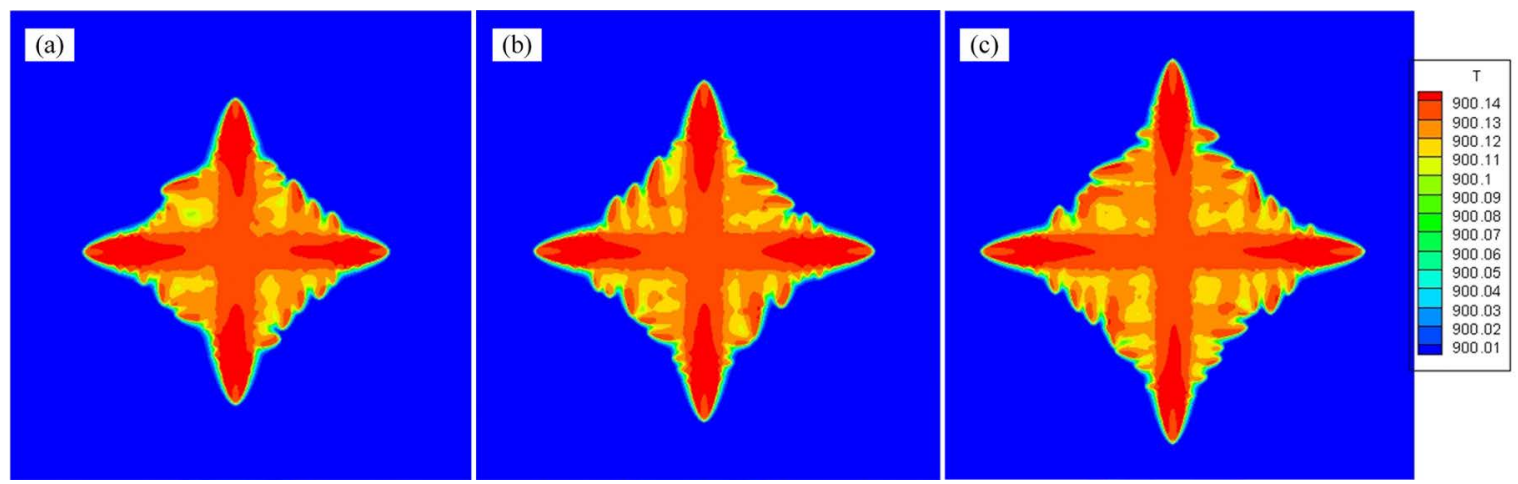

Figure 6. Temperature distribution in an undercooled melt of Al-Cu binary alloy with different anisotropies after $4.7 \times 10^{-5} \mathrm{~s}$ : (a) 0.03 , (b) 0.04 , and (c) 0.05 


\section{Conclusion}

1. A phase field model is proposed to investigate the dendritic growth of Al-Cu binary alloy during the non-isothermal solidification process by coupling the phase field method, solute diffusion and temperature transfer.

2. During the non-isothermal solidification process of Al-4.5\% Cu binary alloy, the dendrite grow into undercooled melt with the solute rejection and latent heat release at the front of solid/liquid interface, and the solute enriches at the dendrite root and high temperature appears at the dendritic growth front.

3. The perturbation intensity and anisotropy have great effects on the dendritic growth of $\mathrm{Al}-4.5 \% \mathrm{Cu}$ binary alloy. With the increase of perturbation intensity and anisotropy, the dendrite grows with more developed, and the tertiary dendrite arm appears for the high perturbation intensity and anisotropy. But, the perturbation intensity and anisotropy do not have a big effect on the concentration field and temperature field, the solute is still enriched at the root of dendrite and the high temperature also appears at the dendritic growth front.

\section{Acknowledgements}

The authors gratefully acknowledge the financial support of National Key Research and Development Plan (No. 2017YFB0304100, 2016YFB0300105), National Natural Science of China (No. 51674072, 51704151, 51804067) and Fundamental Research Funds for the Central Universities (No. N182504014, N170708020, N172503013).

\section{References}

1. Yasuda H, Nagira T, Yoshiya M, Nakatsuka N, Sugiyama A, Uesugi K, et al. Development of X-ray Imaging for Observing Solidification of Carbon Steels. ISIJ International. 2011;51(3):402408.

2. Kobayashi R. Modeling and numerical simulations of dendritic crystal growth. Physica D: Nonlinear Phenomena. 1993;63(34):410-423.
3. Fife PC, Gill GS. The phase-field description of mushy zones. Physica D: Nonlinear Phenomena. 1989;35(1-2):267-275.

4. Weeler AA, Boettinger WJ, McFadden GB. Phase-field model for isothermal phase transition in binary alloys. Physical Review A. 1992;45(10):7424-7439.

5. Wheeler AA, Murray BT, Schaefer RJ. Computation of dendrites using a phase field model. Physica D: Nonlinear Phenomena. 1993;66(1-2):243-262.

6. Wheeler AA, Ahmad NA, Boettinger WJ, Braun RJ, McFadden GB, Murray BT. Recent developments in phase-field models of solidification. Advances in Space Research. 1995;16(7):163127.

7. Thiessen RG, Sietsma J, Palmer TA, Elmer JW, Richardson IM. Phase-field modelling and synchrotron validation of phase transformations in martensitic dual-phase steel. Acta Materialia. 2007;55(2):601-614.

8. Boettinger WJ, Warren JA. The phase-field method: simulation of alloy dendritic solidification during recalescence. Metallurgical and Materials Transactions A. 1996;27(3):657-669.

9. Kobayashi H, Ode M, Kim SG, Kim WT, Suzuki T. Phasefield model for solidification of ternary alloys coupled with thermodynamic database. Scripta Materialia. 2003;48(6):689694.

10. Suzuki T, Ode M, Kim SG, Kim WT. Phase-field model of dendritic growth. Journal of Crystal Growth. 2002;237-239(Pt 1):125-131.

11. Kim SG, Kim WT, Suzuki T. Phase-field model for binary alloys. Physical Review: E. 1999;60(6 Pt B):7186-7197.

12. Kim SG, Kim WT. Phase-field modeling of rapid solidification. Materials Science and Engineering: A. 2001;304-306:281-286.

13. Ode M, Suzuki T. Numerical Simulation of Initial Microstructure evolution of Fe-C Alloys Using a Phase-Field Model. ISIJ International. 2002;42(4):368-374.

14. Ode M, Suzuki T, Kim SG, Kim KT. Phase-field model for solidification of Fe-C alloys. Science and Technology of Advanced Materials. 2000;1(1):43-49.

15. Zhang Y, Li D, Wang C, Li Y. Simulation of dendrite growth of Fe-C alloy using phase field method. Chinese Journal of Materials Research. 2009;23(3):317-322.

16. Niu YE, Yan W, Feng XM, Chen J, Fan XH. Phase-field simulation of Fe-C alloy in the isothermal solidification. Foundry Technology. 2008;29:244-249. 